\title{
SISTEMATIZACIÓN DE LA IMPLEMENTACIÓN \\ DEL SISTEMA DE INGRESO PRIORITARIO DE \\ EQUIDAD EDUCATIVA (EXCUPO DE EQUIDAD) \\ EN LA CARRERA DE PSICOLOGÍA DE LA UNIVERSIDAD DE CHILE 2010-2012
}

\section{Bases empíricas y teóricas de la experiencia}

Durante el 2009 el claustro académico del Departamento de Psicología de la Universidad de Chile decidió implementar una acción afirmativa denominada "cupo de equidad", que buscó favorecer el ingreso de estudiantes de los tres primeros quintiles y de instituciones de enseñanza media municipal.

Esta decisión se fundamentó en la evidencia empírica que da cuenta de las desigualdades en el acceso a la educación superior en Chile (Espinoza y González, 2011; Díaz-Romero, 2010; Javier Gil, 2010) y en la propia universidad, la que al compararse con el resto de instituciones del Consejo de Rectores (CRUCH) ocupa el segundo lugar nacional en contribuir en acentuar la inequidad del sistema, ya que no más del $27 \%$ de su matrícula proviene del sector de menores ingresos económicos (OCDE, 2009), y solo uno de cada cinco estudiantes son egresados de liceos municipales (Comisión Equidad Universidad de Chile, 2011). A su vez la carrera de Psicología de esta universidad no era una excepción, ya que en función de la información interna disponible se podía observar una disminución sostenida de estudiantes provenientes del sector municipal de la educación desde el 2003 y hasta el 2009, según así se aprecia a continuación (Gráfico 1): 
Gráfico 1: Tendencia de matrícula según tipo de establecimiento de egreso

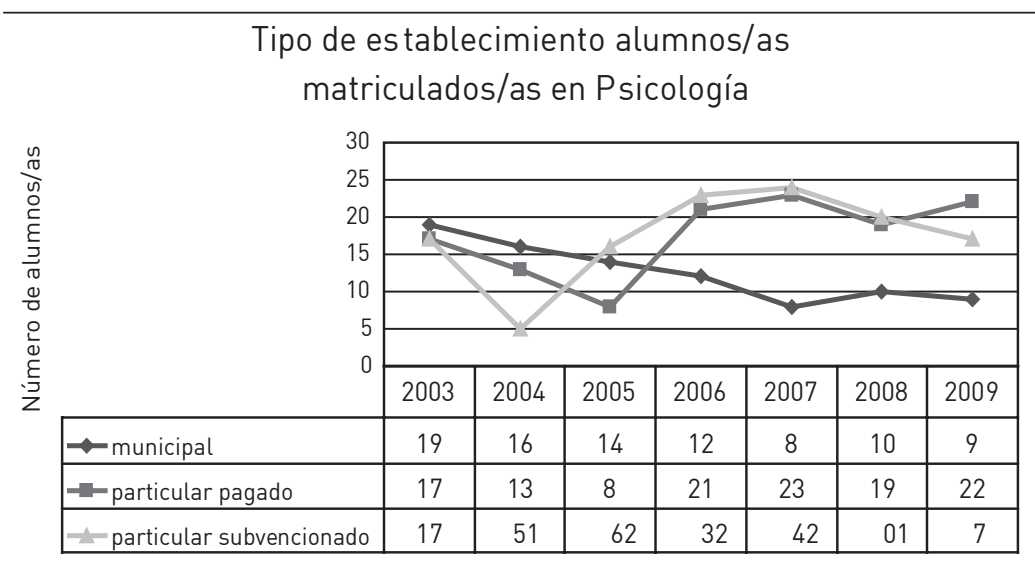

Fuente: Dirección Académica Facultad de Ciencias Sociales (2009).

La noción de equidad desde una perspectiva marxista promovería la igualdad desde la máxima "a cada cual según su capacidad, y cada cual según sus necesidades" (Fetscher, 1988, p. 187, en Neves de Azevedo, 2013, p. 136), reconociendo que en sociedades capitalistas es imposible el desarrollo de una igualdad sustantiva, la que sería accesible solo en un escenario pos revolucionario, cuyas relaciones de producción sean coherentes con dicha igualdad (Neves de Azevedo, 2013). Así, siguiendo a Giroux (1985), las acciones afirmativas y las mismas universidades que las implementan se debaten entre la reproducción de las desigualdades de clase y la resistencia a ellas.

Para la correcta concreción de las estrategias de acceso y permanencia en la educación superior se consideró atender a las necesidades, capacidades y logros de los estudiantes en las distintas dimensiones que componen la trayectoria universitaria, es decir, el acceso, la permanencia, los logros y los resultados de todos los estudiantes (Espinoza, González y Latorre, 2009). Se tomó también en cuenta el modelo de interacción (Tinto, 1989) que explica el proceso de permanencia universitaria como el grado de ajuste entre el estudiante y la institución, producto de las experiencias académicas 
340 SISTEMATIZACIÓN DE LA IMPLEMENTACIÓN DEL SISTEMA DE INGRESO PRIORITARIO DE EQUIDAD EDUCATIVA (EXCUPO DE EQUIDAD) EN LA CARRERA DE PSICOLOGÍA DE LA UNIVERSIDAD DE CHILE 2010-2012 - M. Castro, C. Aranda, C. Castro, H. de Torres, C. Lizama, J. Williams

y sociales (integración). Finalmente, desde una perspectiva inclusiva, se optó por un enfoque que supone una transformación global institucional y de lo que ocurre en el aula en pos del aprendizaje y participación de todos los estudiantes en un ambiente de diversidad (Coll y Miras, 2001).

\section{Descripción de la experiencia}

De modo concreto el 2009 se definieron los siguientes criterios para postular al cupo de equidad:

- Ingreso familiar dentro de los tres primeros quintiles.

- Cursar la enseñanza media completa en un establecimiento municipal.

- Postular a beneficios económicos del Estado.

- Postular el mismo año de egreso de la enseñanza media y en primera preferencia a la carrera de Psicología de la Universidad de Chile.

- Obtener 600 puntos ponderados en la PSU².

El proceso de postulación implicó una primera etapa en la que los postulantes acreditaron los cuatro primeros criterios, previamente a rendir la PSU, siendo el puntaje en esta prueba de selección y la correcta postulación a la carrera los últimos criterios que se debían cumplir.

Finalmente, para seleccionar a los sujetos la Comisión de Docencia de la carrera de Sociología ${ }^{3}$ elaboró una propuesta de criterios que ponía en cuestión el utilizar el puntaje ponderado de la PSU como criterio final de selección y jerarquización de quienes podían matricularse. Por lo que se propusieron tres variables para priorizar perfiles. Estas son, en orden de relevancia al momento de elaborar las jerarquías:

2 La ponderación PSU en ese momento para Psicología correspondía a 30\% de las notas de enseñanza media, 30\% en la prueba de lenguaje y comunicación, 25\% en la prueba de matemáticas y $15 \%$ en la prueba específica de historia y ciencias sociales o ciencias, ya que es obligación por lo menos rendir una de estas dos últimas.

3 Carrera que se suma a la experiencia el 2011. 
- Quintil de ingreso: pertenecer al quintil 1,2 o 3, priorizando en ese orden.

- Ranking de egreso: priorizar a quienes estén dentro del 10\% mejor de su generación de egreso del colegio.

- Índice de vulnerabilidad económica: priorizar a quienes provengan de colegios con 50 o más puntos en este índice, pues indica mayor vulnerabilidad.

Así, se privilegió a aquellos postulantes del quintil 1, que eran el 10\% mejor en términos de ranking de egreso y que provinieran de colegios más vulnerables.

Para impactar en las dimensiones de logro y permanencia se desarrolló como estrategia de adaptación de la institución al aumento en la diversidad, la creación de un grupo de colaboración que operaba dentro de los dos primeros años de la carrera (ciclo básico). Esta estructura, dependiente de la jefatura de carrera y a su vez de la coordinación de ciclo básico, se denominó "equipo de apoyo a la innovación", y sus ejes de trabajo fueron: a) apoyo a la innovación de las prácticas docentes; b) apoyo a estudiantes; y c) gestión de la información para la toma de decisiones pedagógicas y administrativas.

El objetivo de este equipo fue desarrollar acciones articuladas que buscaron promover, posibilitar y facilitar el acceso a una educación de calidad, la permanencia en el sistema, y el logro de aprendizajes de todos/as los/as alumnos/as durante su paso por el Ciclo Inicial de Formación de Psicología. A su vez, los principios básicos que guiaron el trabajo fueron los siguientes: agenciamiento, en cuanto se considera la acción de los sujetos en sus propias trayectorias como trascendental (Giroux, 1985); voluntariedad en la participación de actividades de apoyo; confianza, considerando la relevancia de la cercanía con los sujetos con quienes se trabaja; y colaboración, para promover una lógica de trabajo en red.

El eje de apoyo a la innovación de las prácticas docentes se constituyó desde una perspectiva de asesoramiento, y sus actividades más importantes fueron: apoyo a la planificación de nuevas prácticas pedagógicas; revisión de la metodología de enseñanza y evaluación de aprendizajes; apoyo en la elaboración de programas de asignaturas; 

PRIORITARIO DE EQUIDAD EDUCATIVA (EXCUPO DE EQUIDAD) EN LA CARRERA DE PSICOLOGÍA DE LA UNIVERSIDAD DE CHILE 2010-2012 - M. Castro, C. Aranda, C. Castro, H. de Torres, C. Lizama, J. Williams

colaboración en la formación pedagógica de estudiantes ayudantes y coordinación con unidades institucionales vinculadas con el desarrollo docente.

El eje de apoyo a estudiantes se orientó hacia la colaboración en los procesos de inducción universitaria, como también en el logro y la permanencia de todos los estudiantes del ciclo inicial. Las tareas implicadas fueron: caracterización de los/as estudiantes, en coordinación con línea de gestión de la información; propuestas y organización del proceso de inducción a la facultad y la carrera; coordinación, realización (de ser necesario) y sistematización de intervenciones para la mejora en los hábitos de estudio u otros cursos que respondan a las necesidades de los estudiantes; dinamización y seguimiento al acompañamiento a estudiantes de primer año por parte de sus compañeros/as de los años superiores; trabajo en red con servicios institucionales; indagación en las problemáticas que pudiesen perjudicar a los/as estudiantes en su desempeño, apoyando y gestionando la búsqueda de soluciones al respecto.

El eje de gestión de la información buscó la creación de un sistema de información que apoyara la administración y seguimiento de información pedagógica curricular del Ciclo Inicial de Formación para lograr un flujo eficaz de información y llevar a cabo los procesos de apoyo. La tareas implicadas fueron: sistematización de la información recopilada producto de las diversas actividades realizadas; confección de bases de datos; caracterización de estudiantes; sistematización de procedimientos y acuerdos de trabajo con unidades relacionadas con gestión de la información.

\section{Descripción de resultados}

Para realizar un seguimiento de esta experiencia se consideraron los siguientes aspectos: la composición de la matrícula de primer año de acuerdo con el tipo de establecimiento del cual provenían los alumnos; los indicadores de permanencia en el sistema; el logro académico; y la valoración que los estudiantes del ciclo básico de Psicología realizaron sobre las labores llevadas a cabo por el equipo de innovación. 


\subsection{Composición de la matrícula primer año (Tabla 1)}

Tabla 1: Composición matrícula 1er año 2010, 2011, 2012

\begin{tabular}{|c|c|c|c|c|c|c|}
\hline \multirow[b]{2}{*}{ Año ingreso } & \multirow[b]{2}{*}{ Dependencia } & \multicolumn{5}{|c|}{ Vía de ingreso* } \\
\hline & & PSU & Equidad & $\mathrm{BEA}^{4}$ & Otra vía & Total \\
\hline \multirow{5}{*}{2010} & Municipal & 6 & 18 & 3 & 0 & 27 \\
\hline & Particular subvencionado & 18 & 0 & 6 & 0 & 24 \\
\hline & Particular pagado & 24 & 0 & 0 & 1 & 25 \\
\hline & Sin información & 3 & 0 & 1 & 0 & 4 \\
\hline & Total & 51 & 18 & 10 & 1 & 80 \\
\hline \multirow{5}{*}{2011} & Municipal & 12 & 23 & 5 & 0 & 40 \\
\hline & Particular subvencionado & 21 & 0 & 8 & 2 & 31 \\
\hline & Particular pagado & 19 & 0 & 0 & 0 & 19 \\
\hline & Sin información & 4 & 0 & 3 & 0 & 7 \\
\hline & Total & 56 & 23 & 16 & 2 & 97 \\
\hline \multirow{5}{*}{2012} & Municipal & 6 & 20 & 4 & 1 & 31 \\
\hline & Particular subvencionado & 26 & 0 & 12 & 0 & 38 \\
\hline & Particular pagado & 17 & 0 & 0 & 1 & 18 \\
\hline & Sin información & 11 & 0 & 2 & 0 & 13 \\
\hline & Total & 60 & 20 & 18 & 2 & 100 \\
\hline
\end{tabular}

* No se consideran extranjeros.

Fuente: Comisión Equidad FACSO (2010-2011).

Se aprecia que para cada año el cupo de equidad ha significado la fuente más importante de estudiantes provenientes del sector municipal. Si bien se quiebra la tendencia a la disminución de estudiantes de este sector -que se apreciaba desde el 2003-, solo en el 2010 de implementación se logra una distribución de tres tercios de acuerdo con el tipo de dependencia administrativa del cual provienen los/as estudiantes matriculados/as. Hay que considerar en todo caso para el 2012 que no se cuenta con información específica del tipo de establecimiento del cual proviene una importante cantidad de sujetos.

$4 \quad$ BEA corresponde a Beca de Excelencia Académica. 
344 SISTEMATIZACIÓN DE LA IMPLEMENTACIÓN DEL SISTEMA DE INGRESO PRIORITARIO DE EQUIDAD EDUCATIVA (EXCUPO DE EQUIDAD) EN LA CARRERA DE PSICOLOGÍA DE LA UNIVERSIDAD DE CHILE 2010-2012 - M. Castro, C. Aranda, C. Castro, H. de Torres, C. Lizama, J. Williams

\subsection{Permanencia en el sistema (Tabla 2)}

Tabla 2: Renuncias y postergaciones según vía de ingreso

\begin{tabular}{c|l|c|c|c|c}
\hline \multirow{2}{*}{ Año* } & \multicolumn{1}{|c|}{ Situación } & PSU & Equidad & Otro & Total \\
\hline \multirow{2}{*}{2010} & Posterga2 & 2 & 0 & 0 & 2 \\
\cline { 2 - 6 } & Renuncia3 & 1 & 0 & 0 & 1 \\
\hline \multirow{2}{*}{2011} & Posterga & 1 & 0 & 0 & 1 \\
\cline { 2 - 6 } & Renuncia & 0 & 1 & 1 & 2 \\
\hline \multirow{2}{*}{2012} & Posterga & 1 & 5 & 0 & 6 \\
\cline { 2 - 6 } & Renuncia & 5 & 0 & 1 & 6 \\
\hline & Total & 10 & 6 & 2 & 18 \\
\hline
\end{tabular}

*Año en que el estudiante presenta la solicitud.

Fuente: elaboración propia a base de informe de Secretaría de Estudios, FACSO (2012).

La información actualizada al primer semestre del 2012 muestra que entre el 2010 y 2012 del total de estudiantes matriculados vía PSU, cuatro estudiantes han postergado estudios y seis han renunciado a la carrera. En el caso de aquellos ingresados vía equidad, han postergado cinco y solo un estudiante ha renunciado. Luego de analizar las razones tanto de postergación y como de renuncia, destaca el hecho de que las primeras tienden a aludir a problemas de salud y las segundass a razones vocacionales. Ningún estudiante ha sido eliminado por razones académicas.

\subsection{Logro académico}

Tabla 3: Resultados de comparación logro académico según vía de ingreso

\begin{tabular}{c|c|c|c|c|c|c|}
\hline \multicolumn{7}{|c|}{ ANOVA de un factor para vía de ingreso y promedio semestral } \\
\hline & \multicolumn{7}{|c|}{ Promedio final } & \multicolumn{2}{c|}{ ANOVA } \\
\hline Semestre & Equidad & PSU & BEA & Estadístico de Levene* & F & Sig. \\
\hline I & 5,245 & 5,53 & 5,369 & $0,577(0,563)$ & 8,795 & 0 \\
\hline II & 4,946 & 5,374 & 5,325 & $0,498(0,608)$ & 6,783 & 0,002 \\
\hline III & 5,337 & 5,666 & 5,502 & $0,282(0,755)$ & 5,77 & 0,004 \\
\hline IV & 5,122 & 5,665 & 5,528 & $2,407(0,099)$ & 5,507 & 0,006 \\
\hline V & 6,016 & 6,051 & 6,39 & $1,19(0,312)$ & 4,136 & 0,021 \\
\hline
\end{tabular}

Solicitud de interrumpir los estudios por un tiempo acotado.

Abandono definitivo del programa. 
Tabla 4: Comparaciones múltiples del logro académico según vía de ingreso

\begin{tabular}{|c|c|c|c|c|c|c|c|}
\hline \multicolumn{8}{|c|}{ Comparaciones múltiples } \\
\hline \multirow[b]{2}{*}{$\begin{array}{l}\text { Promedios } \\
\text { finales }\end{array}$} & \multirow[b]{2}{*}{$\begin{array}{l}\text { (I) Vía de } \\
\text { ingreso } \\
\end{array}$} & \multirow[b]{2}{*}{$\begin{array}{c}\text { (J) Vía de } \\
\text { ingreso }\end{array}$} & \multirow[b]{2}{*}{$\begin{array}{l}\text { Diferencia de } \\
\text { medias (I-J) }\end{array}$} & \multirow[b]{2}{*}{$\begin{array}{l}\text { Error } \\
\text { típico }\end{array}$} & \multirow[b]{2}{*}{ Sig. } & \multicolumn{2}{|c|}{$\begin{array}{c}\text { Intervalo de confianza } \\
\text { al } 95 \% \\
\end{array}$} \\
\hline & & & & & & $\begin{array}{l}\text { Límite } \\
\text { inferior }\end{array}$ & $\begin{array}{l}\text { Límite } \\
\text { superior }\end{array}$ \\
\hline \multirow{6}{*}{$\begin{array}{l}\text { Primer } \\
\text { semestre }\end{array}$} & \multirow{2}{*}{ BEA } & Equidad & 0,12404 & 0,09341 & 0,382 & $-0,0969$ & 0,345 \\
\hline & & PSU & $-0,1602$ & 0,08188 & 0,127 & $-0,3539$ & 0,0335 \\
\hline & \multirow{2}{*}{ Equidad } & BEA & $-0,12404$ & 0,09341 & 0,382 & $-0,345$ & 0,0969 \\
\hline & & PSU &,$- 28424^{*}$ & 0,06967 & 0 & $-0,4491$ & $-0,1194$ \\
\hline & \multirow{2}{*}{ PSU } & BEA & 0,1602 & 0,08188 & 0,127 & $-0,0335$ & 0,3539 \\
\hline & & Equidad &, $28424^{*}$ & 0,06967 & 0 & 0,1194 & 0,4491 \\
\hline \multirow{6}{*}{$\begin{array}{l}\text { Segundo } \\
\text { semestre }\end{array}$} & \multirow{2}{*}{ BEA } & Equidad & $.37885^{*}$ & 0,15915 & 0,048 & 0,0021 & 0,7556 \\
\hline & & PSU & $-0,04934$ & 0,14109 & 0,935 & $-0,3834$ & 0,2847 \\
\hline & \multirow{2}{*}{ Equidad } & BEA &,$- 37885^{*}$ & 0,15915 & 0,048 & $-0,7556$ & $-0,0021$ \\
\hline & & PSU &,$- 42819 *$ & 0,1178 & 0,001 & $-0,7071$ & $-0,1493$ \\
\hline & \multirow{2}{*}{ PSU } & BEA & 0,04934 & 0,14109 & 0,935 & $-0,2847$ & 0,3834 \\
\hline & & Equidad & $.42819 *$ & 0,1178 & 0,001 & 0,1493 & 0,7071 \\
\hline \multirow{6}{*}{$\begin{array}{l}\text { Tercer } \\
\text { semestre }\end{array}$} & \multirow{2}{*}{ BEA } & Equidad & 0,16513 & 0,13509 & 0,442 & $-0,1549$ & 0,4852 \\
\hline & & PSU & $-0,16429$ & 0,11847 & 0,351 & $-0,445$ & 0,1164 \\
\hline & \multirow{2}{*}{ Equidad } & BEA & $-0,16513$ & 0,13509 & 0,442 & $-0,4852$ & 0,1549 \\
\hline & & PSU &,$- 32941^{*}$ & 0,0986 & 0,003 & $-0,563$ & $-0,0958$ \\
\hline & \multirow{2}{*}{ PSU } & BEA & 0,16429 & 0,11847 & 0,351 & $-0,1164$ & 0,445 \\
\hline & & Equidad &, $32941^{*}$ & 0,0986 & 0,003 & 0,0958 & 0,563 \\
\hline \multirow{6}{*}{$\begin{array}{l}\text { Cuarto } \\
\text { semestre }\end{array}$} & \multirow{2}{*}{ BEA } & Equidad & 0,40611 & 0,21376 & 0,148 & $-0,1076$ & 0,9198 \\
\hline & & PSU & $-0,13752$ & 0,17607 & 0,716 & $-0,5607$ & 0,2856 \\
\hline & \multirow{2}{*}{ Equidad } & BEA & $-0,40611$ & 0,21376 & 0,148 & $-0,9198$ & 0,1076 \\
\hline & & PSU &,$- 54363^{*}$ & 0,16385 & 0,004 & $-0,9374$ & $-0,1499$ \\
\hline & \multirow{2}{*}{ PSU } & BEA & 0,13752 & 0,17607 & 0,716 & $-0,2856$ & 0,5607 \\
\hline & & Equidad & $.54363^{*}$ & 0,16385 & 0,004 & 0,1499 & 0,9374 \\
\hline \multirow{6}{*}{$\begin{array}{l}\text { Quinto } \\
\text { semestre }\end{array}$} & \multirow{2}{*}{ BEA } & Equidad & $.37400^{*}$ & 0,15061 & 0,042 & 0,0112 & 0,7368 \\
\hline & & PSU &, $33850^{*}$ & 0,12297 & 0,021 & 0,0423 & 0,6347 \\
\hline & \multirow{2}{*}{ Equidad } & BEA &,$- 37400^{*}$ & 0,15061 & 0,042 & $-0,7368$ & $-0,0112$ \\
\hline & & PSU & $-0,0355$ & 0,11226 & 0,946 & $-0,3059$ & 0,2349 \\
\hline & \multirow{2}{*}{ PSU } & BEA &,$- 33850^{*}$ & 0,12297 & 0,021 & $-0,6347$ & $-0,0423$ \\
\hline & & Equidad & 0,0355 & 0,11226 & 0,946 & $-0,2349$ & 0,3059 \\
\hline
\end{tabular}

* La diferencia de medias es significativa al nivel 0,05.

A partir del análisis de varianza de un factor para las vías de ingreso se observaría que existen diferencias significativas para todos los semestres entre los grupos ( $\mathrm{Sig}<0,05)$. No obstante, al ejecutar la prueba Post-Hoc HSD de Tukey (E. de Levene $>0,05$ ) se aprecia que las diferencias que históricamente aparecen como significativas entre PSU y Equidad durante los primeros cuatro semestres, desaparecen 
346 SISTEMATIZACIÓN DE LA IMPLEMENTACIÓN DEL SISTEMA DE INGRESO PRIORITARIO DE EQUIDAD EDUCATIVA (EXCUPO DE EQUIDAD) EN LA CARRERA DE PSICOLOGÍA DE LA UNIVERSIDAD DE CHILE 2010-2012 - M. Castro, C. Aranda, C. Castro, H. de Torres, C. Lizama, J. Williams

en el quinto. Por tanto, la diferencia significativa de este último semestre se debe a que el grupo BEA obtiene un rendimiento superior a los otros dos grupos y supera al grupo PSU de manera significativa (Sig. =0,021).

\subsection{Valoración estudiantil de trabajo realizado por equipo de apoyo a la innovación}

Para complementar los indicadores anteriores, así como incorporar la visión que los/as alumnos/as han tenido del trabajo realizado por el equipo, se procedió a consultar de forma anónima a todos los/as estudiantes matriculados/as en el 2010, 2011 y 2012 por los aspectos positivos y a aquellos por mejorar del trabajo de apoyo.

Para analizar las respuestas se trabajó desde el análisis de contenido cualitativo (Abela, 2003), se definieron categorías de análisis inductivamente, las que con posterioridad se agruparon en función del eje de trabajo al cual referían.

\subsubsection{Apoyo a estudiantes}

Se aprecia que de modo general se valora antes que todo la ayuda que otorga el equipo a los estudiantes en su integración y acercamiento tanto a la carrera como a sus compañeros, la información que se entrega y la forma cómo se enfocan las funciones de apoyo. Así se expresa en la siguiente cita:

La gran ayuda prestada para con nosotros al enfrentar nuestro primer año académico en la universidad. La buena disposición para responder todas nuestras dudas respecto de ramos, la carrera, etc., que nos permite sobrellevar de mejor manera nuestra estadía en nuestro primer año.

Por otra parte, al revisar los elementos que los estudiantes consideran son necesarios de mejorar, destacan los canales de comunicación entre los estudiantes y el equipo, añadiendo que les gustaría que se establecieran contactos en persona, ya que manifiestan que algunos estudiantes no saben cómo dirigirse al equipo. Ejemplos de esta categoría son los siguientes: 
A pesar de que el sistema por internet sea efectivo y bueno, no estaría mal algo más personalizado.

Mejoraría el tema de la comunicación con el equipo de innovación, ya que a veces muchos estudiantes quieren obtener información o tienen dudas respecto de ciertos temas y no saben a dónde dirigirse o con quién hablar al respecto.

Junto con lo anterior, destaca la petición de información como la entrega de orientaciones acerca del campus y la facultad antes de ingresar a la carrera, en especial para estudiantes que no son de la capital. Lo anterior se expresa en opiniones de los estudiantes tales como:

Antes de iniciar con las actividades de integración a la carrera, den formas o rutas más fáciles para llegar a la facultad, a los correos de los mechones, eso dificulta mucho a quienes viven lejos o fuera de Santiago.

Al mismo tiempo, los estudiantes manifiestan requerir mayor cercanía por parte del equipo, donde este generase instancias de participación y diálogo con el estudiantado, que facilite la aparición de demandas. Los jóvenes lo explicitan de la siguiente forma:

Generaría más instancias donde se pudiera participar con el equipo, ya sea con cosas informativas o de apoyo, que integren a todos, que no solo se dé en una instancia que haya que acercarse. A veces uno no genera dudas de algo que desconocemos.

Existe la preocupación respecto de la integración de los compañeros que ingresan por cupo de equidad y que no pertenecen a liceos emblemáticos. A continuación se presenta un ejemplo:

Cosas a mejorar serían darle una continuidad al apoyo brindado a los cupos de equidad, específicamente a aquellos alumnos provenientes de colegios y liceos no emblemáticos, porque pareciera ser que los alumnos que salen de colegios emblemáticos y hacen uso de los cupos de equidad son muchos y llegan a la universidad con lazos interpersonales ya creados en el colegio, haciendo sentir a aquellos de colegios no emblemáticos una persona sin lazos interpersonales en la universidad. 

PRIORITARIO DE EQUIDAD EDUCATIVA (EXCUPO DE EQUIDAD) EN LA CARRERA DE PSICOLOGÍA DE LA UNIVERSIDAD DE CHILE 2010-2012 - M. Castro, C. Aranda, C. Castro, H. de Torres, C. Lizama, J. Williams

También sobre la mejora de las ayudas entregadas por el equipo se indica la necesidad que se detecte a tiempo los estudiantes con alguna dificultad en la carrera. Como ejemplo de lo antes mencionado, los jóvenes plantean:

Me gustaría que hubiese más instancias donde se diera la interacción innovación-alumnado, más allá de las charlas en la semana de inducción de los mechones. Lamentablemente les pasa seguido que se encuentran con el caso de algún alumno que reprobó muchos ramos o que debe irse de la carrera y para entonces no se puede hacer nada. Dependen demasiado de que el alumno se decida a buscar ayuda y no muchos lo hacen. Me gustaría que hubiese instancias en las que se pudiera detectar a aquellos que por desinformación, temor o recelo, no se acercan en busca de ayuda y tratan (a veces sin éxito) de bancárselas solos.

Por último, los estudiantes manifiestan que les gustaría que se generaran diversos talleres como hábitos de estudio, manejo de estrés, etc. En relación con esto los estudiantes plantean:

Me gustaría que organizaran talleres para nivelar aspectos educacionales: elaboración de ensayos, técnicas de estudio, enfrentamiento del estrés, expresión corporal (para las presentaciones), utilización efectiva de audiovisuales (ppt).

\subsubsection{Apoyo a innovación de prácticas pedagógicas, visión de los estudiantes}

Los estudiantes valoran positivamente la generación de diversos materiales y actividades utilizados tanto en las clases como en las evaluaciones. Según sus apreciaciones esto significa para ellos una preocupación por su aprendizaje, lo que también le da mayor dinamismo y organización a las actividades de la cátedra. Ante la consulta ¿qué elementos valora positivamente?, consignan lo siguiente:

Hacen menos monótono el aprendizaje.

La preocupación por la mejora en la docencia en cuanto a material de estudio y apoyo académico.

Las pautas y rúbricas que han desarrollado en los ramos.

La ayuda y mejora de varios Power Point utilizados durante la cátedra de ciertos ramos. 
A partir del mismo aspecto se percibe positivamente que estas actividades y materiales fomenten la participación de los estudiantes y el trabajo en grupo.

Han trabajado en ciertos ramos con proyectos innovadores que apuntan a dinámicas grupales que me parecen muy positivas. Me gustó el rol más participativo que se dio a los estudiantes, se agradecen las metodologías más dinámicas.

Asimismo, ante la pregunta acerca de qué elementos mejoraría, en este aspecto se solicita la intervención del equipo en ramos en donde ellos perciben algunas dificultades en la docencia, como también resaltan la necesidad de evaluar las innovaciones durante la asignatura y no al final, con el fin de movilizar cambios cuando ellos cursan los ramos:

Mejorar la calidad y dinámica de todos los ramos, no solo uno. Trabajar en conjunto con todos los profesores para ver sus falencias. Realizar encuestas u otro tipo de método de acopio de datos durante el semestre, para así realizar cambios en el transcurso de las cátedras, no una vez ya finalizadas.

Otro aspecto que cabe destacar es la percepción de algunas de las intervenciones realizadas en clases y evaluaciones. Esto, ya que ante la consulta respecto de qué mejorarían, relatan que muchas de esas actividades son "infantiles", o que falta consultar a los estudiantes acerca de estas mismas innovaciones, las cuales a veces son recibidas de manera negativa.

Se han vuelto más dinámicas muchas clases, pero falta incorporar la visión de los alumnos, ya que a veces por innovar los cursos se desordenan. Asimismo, ha fomentado una modificación y adaptación de la carrera, lo que es bueno.

Las actividades que se hacen, por su carácter innovador, cuesta que sean recibidas por los estudiantes, sobre todo al momento de las evaluaciones, en donde uno está acostumbrado a actividades/evaluaciones más clásicas. Tal vez innovar en clases y no en las evaluaciones enganche a más, pero no hacer cosas infantiles. 

PRIORITARIO DE EQUIDAD EDUCATIVA (EXCUPO DE EQUIDAD) EN LA CARRERA DE PSICOLOGÍA DE LA UNIVERSIDAD DE CHILE 2010-2012 - M. Castro, C. Aranda, C. Castro, H. de Torres, C. Lizama, J. Williams

Uno de los elementos que mencionan los estudiantes para mejorar el trabajo del equipo en su área de apoyo a docentes es que exista una mejor integración y coordinación entre las actividades y los objetivos y evaluaciones de las asignaturas.

Principalmente mejoraría la vinculación entre las metodologías propuestas por innovación y la evaluación de estas por parte del equipo docente. Generar un trabajo con mayor desarrollo con el equipo docente, para poder proponer metodologías que puedan dar cuenta de los objetivos de la asignatura.

La preocupación por tener mayor incidencia y ser parte activa del proceso de innovación es algo que no aparece de igual manera en todas las generaciones. Se podría decir que esta preocupación no aparece en el primer y segundo año. Es recién en la generación 2010 donde se encuentran opiniones como esta:

Antes de decidir implementar alguna innovación, yo realizaría pequeñas instancias que permitan conocer la necesidad de los alumnos de la "innovación", disposición ante el trabajo y, finalmente, incluir a alumnos en la elaboración de los proyectos.

\section{Conclusiones}

Es posible apreciar que esta acción afirmativa permitió corregir aspectos vinculados con el acceso a la carrera, la que mediante el cupo de equidad pudo romper la tendencia que excluía de sus aulas a estudiantes de escasos recursos del sector municipal.

Sobre la permanencia destaca el hecho de que son razones vocacionales las que llevan a sujetos que han ingresado vía PSU a desistir de sus estudios. Por otro lado, la mayoría de quienes ingresaron vía cupo de equidad se mantienen en el programa, y si bien se debe profundizar en la razones de salud que aparecen en sus postergaciones, no surgen eliminaciones por temas académicos, contradiciendo lo que muchos detractores de este tipo de medidas señalan al argumentar en contra de la implementación de acciones afirmativas en la enseñanza superior. 
En el logro académico se observa que los/as estudiantes que ingresan vía equidad se demoran dos años en equiparar sus calificaciones a las de sus pares, lo que ha sido ya descrito en la experiencia del Propedéutico de la Universidad de Santiago (Castillo, Cabezas, 2010). Esto pone en evidencia las limitaciones en esta mutua adaptación entre institución y estudiantes, que se expresaría en la reproducción de desigualdades (Neves de Azevedo, 2013), ya sea en los resultados PSU o más tarde en las calificaciones universitarias.

Para los estudiantes resultan visibles y valorables las estrategias de adaptación de la institución y los mecanismos de apoyo creados para favorecer el aprendizaje y participación de todos los estudiantes. Aspectos por mejorar son la proximidad personal de quienes apoyan, así como la capacidad del programa para detectar a tiempo las necesidades de los estudiantes, junto con respetar la autonomía y participación de estos, por ejemplo, en las estrategias de innovación pedagógica para la diversidad.

Finalmente, un impacto importante de esta experiencia es que ha colaborado para que se extienda el tema de equidad a toda la Universidad de Chile, ya que siguiendo el ejemplo de Psicología, y con el importante impulso de la Prorrectoría, a partir del 2013 todas las carreras han abierto cupos bajo el Sistema de Ingreso Prioritario de Equidad Educativa (SIPEE), por tanto, se ha aportado en alguna medida a la mejora de la equidad y calidad del conjunto de la institución.

\section{Referencias}

Abela, J. A. (2003). Las técnicas de análisis de contenido: una revisión actualizada. Departamento de Sociología de la Universidad de Granada. Recuperado el día 10 de marzo de 2013 desde http//public. centrodeestudiosandaluces.es/pdfs/S200103.pdf

Castillo, J. y G. Cabezas (2010). Caracterización de jóvenes de primera generación en educación superior. Nuevas trayectorias hacia la equidad educativa. Revista Calidad en la educación (32), pp. 54-74. 

PSICOLOGÍA DE LA UNIVERSIDAD DE CHILE 2010-2012 - M. Castro, C. Aranda, C. Castro, H. de Torres, C. Lizama, J. Williams

Coll, C. y Miras, M. (2001). Diferencias individuales y atención a la diversidad en el aprendizaje escolar. En A. Marchesi, C. Coll y J. Palacios (Comp.), Desarrollo psicológico y educación, Tomo 2 (pp. 331-353). Madrid: Alianza Editorial.

Comisión Equidad FACSO (2010). Informe interno. Santiago de Chile: Universidad de Chile.

Comisión Equidad Universidad de Chile (2011). Hacia una política de equidad e inclusión en la Universidad de Chile. Informe al Consejo Universitario. Santiago de Chile: Universidad de Chile.

Díaz-Romero, P. (2010). Universidades de calidad: universidades inclusivas. Presentación en el $3^{\text {er }}$ workshop internacional políticas públicas de inclusión a la educación superior. Cátedra UNESCO de Inclusión a la Educación Superior y la Red de Universidades con Propedéuticos UNESCO, noviembre, Santiago de Chile.

Dirección Académica Facultad de Ciencias Sociales Universidad de Chile. (2009). Informe interno de estadísticas. Santiago de Chile.

Espinoza, O. y González, L. (2011). La educación superior en Chile. Revista Pensamiento Universitario, $N^{\circ} 22$, serie documentos: la universidad colombiana ante la reforma de la educación superior, ASCUN, 111-120.

Espinoza, O., González L. y Latorre. C. (2009). Un modelo de equidad para la educación superior: Análisis de su aplicación al caso chileno. Revista de la Educación Superior, XXXVIII (2), (N 150), 97-112.

Gil, F. J. (2010). Fundamentos del proyecto propedéutico. Datos referenciales. Conferencia $1^{\mathrm{er}}$ workshop internacional acceso y equidad a la educación superior - 2008. En: C. Catrileo, C. (2010), Red de programas Propedéutico-UNESCO. Antecedentes preliminares. Resultados iniciales. Programa Propedéutico Bachillerato en Ciencias y Humanidades. Santiago de Chile: Universidad Católica Silva Henríquez.

Giroux, H. (1985). Teorías de la reproducción y la resistencia en la nueva Sociología de la Educación: un análisis crítico. Cuadernos Políticos, (44) pp: 36-65.

Latorre, C., González, L. y Espinoza, O., (2009). Equidad en la educación superior. Santiago de Chile: Fundación Equitas / Editorial Catalonia.

Neves de Azevedo, M. (2013) Igualdade e Equidade: qual é a medida da justiça social? Avaliação. V. 18, (N¹), pp. 129 - 150.

Organización para la Cooperación y el Desarrollo Económico (OCDE) (2009). Revisión de políticas nacionales de educación: la educación superior en Chile. Santiago de Chile: Mineduc. 
Secretaría de Estudios Facultad de Ciencias Sociales de la Universidad de Chile. (2012). Informe interno. Santiago de Chile.

Tinto, V. (1989). Definir la deserción: una cuestión de perspectiva. Revista de Educación Superior, № 71, pp. 33-51.

Recibido: 1/10/2013

Aceptado: 16/05/2014 